\title{
Methodological guide for teaching the implementation of a GPON network
}

\author{
Raúl J. Martelo ${ }^{\# 1}$, Hector Triana ${ }^{\# 2}$, Gustavo Rodríguez ${ }^{\# 3}$ \\ ${ }^{\#}$ Faculty of Engineering, University of Cartagena, Cartagena, Bolívar, Colombia. \\ ${ }^{1}$ rmartelog1@unicartagena.edu.co \\ 2ptb1204@gmail.com \\ 3 grod2145@gmail.com
}

\begin{abstract}
The present work provides a methodological guide for teaching the configuration of the structure of a GPON network for voice and data transmission. The research was of experimental type with practical approach, due to the need to verify technical aspects then to be applied. In the proposed guide, is indicated how there must be realized the connections and configurations of equipments of optical passive network like OLT and ONT/ONU, and of equipments service providers, to give place to a network of last generation who allows to obtain major hardiness and quality of service (QoS). It was concluded that, in the city of Cartagena, it is complex to implement GPON networks due to the limited coverage of this type of network. It is also concluded that the guide has characteristics to be used as a guide in large-scale network implementations.
\end{abstract}

Keyword - GPON, IPTV, VoIP, Web Service

\section{INTRODUCTION}

Telecommunications ploughs to the point of support in today's society, because they ploughs part of to the technological revolution that you have changed the way people communicate, by shortening distances through sending and receiving information instantly. This has implied an exponential growth in the demand for telecommunication services since 2000 [1], and among which the ones with the highest demand have been those with considerable bandwidth, such as: IPTV, video on demand, High-speed Internet, online video games and other existing ones.

Faced with the increase in demand and user needs, networks must keep pace with social and technological changes, which requires increasingly fast and secure transmissions. As a result, costs of updating, maintenance and electricity consumption have risen, which makes necessary new network architectures, both economical, scalable and friendly to the environment. Types of networks such as PON (Passive Optical Network), turn out to be a good choice to transmit high demand services, due to their capacity of bandwidth and scalability [2].

PON, by using optical fiber as a transmission medium, has displaced copper in terrestrial networks due to characteristics such as noise immunity, low production cost, low attenuation (2dB / km versus $7 \mathrm{~dB} / \mathrm{m}$ of copper) and because it requires little space [3]. However, copper networks are still used in telecommunications; by June 2014, the number of subscribers connected to the internet using DSL lines was 144 million, although multimedia services were a burden for them, so they had to use techniques such as UEP (Unequal Error Protection) to ensure the integrity of the data [4].

Also, there are related hybrid networks such as HFC (Hybrid Fiber Coaxial), which have characteristics such as transmission speeds of up to $30 \mathrm{Mbps}$ and are configured in multiple topologies, for example of this are: FiberToTheBuilding, FiberToTheNode, FiberToTheCurb and FiberToTheHome (applies PON architecture). These networks have presented a greater increase in subscriptions to the FTTH (Fiber To The Home) networks in the last decade [5].

Now, a variant of a PON is GPON, which was created by the ITU-T and is used commercially [6]. Supports asymmetric bandwidth with a speed of 1.24 Gbps upstream and 2.5 Gbps downstream; 64 ONUs (Optical Network Unit) can be connected to each fiber optic line, making it a good option to be used in densely populated urban areas [2]; and it uses Time Division Multiple Access (TDMA), to create channels of communication with users and whose use is focused on remote configuration of ONUs, dynamic allocation of bandwidth and collection of statistical data of the operation of the network [7].

To perform the transmission of services in GPON, it is necessary to correctly configure parameters such as: bandwidth, service type and the VLAN as service input to the OLT (Optical Line Terminator). Implementing a GPON can be a complex activity if you do not have specialized knowledge about networks and telecommunications, therefore, it requires time, studies and efforts to test technical and applied aspects about optical networks. In addition, the existing documentation on the understanding, configuration and maintenance of GPON, has a specialized approach to the network and telecommunications community through the use of conceptual schemes, terminology and explanations that require extensive prior reading, for example, [8] demands a reader with extensive knowledge about PON networks, for the understanding of their official texts 
about the management of the ONT, as the application of recommendations and guidelines under the standard G.984.4.

It should be noted that details such as the above are presented in multiple branches of study and technical processes, and for which methodological guides emerge as guidance, tools of a sequential nature, consisting of steps focused on giving specific details to achieve objectives (FMIN, n.d.). These tools have been applied in various fields, as in [9], who use the previous tool to design a strategic IT plan in IES, which allowed determining stages of the implementation of PEIs and executing strategies to control the procedures performed. Also in the field of ICTs, methodological guides have been used, as is the case of the study by [10], in which with the help of Softprosp software, they proposed an approach through a methodological guide to define the professional profile of academic programs.

Therefore, in the present investigation a methodological guide was created and used as a tool to teach the implementation of a GPON network that transmits multiple services, in order to provide detailed explanation about the configuration of the network and parameters that it uses according to the services to be transmitted. To validate the guide, ZTE company equipment was used; tests were carried out with the transmission of IPTV, VoIP and Web Service services.

\section{Methodology}

Experimental research with a practical approach was used, because it was necessary to check technical aspects such as QoS, so that they could be applied.

For the elaboration of the guide, on-site technical visits were made in the facilities of the SENA (National Educational Learning Service), in the city of Cartagena, department of Bolívar, Colombia, in order to carry out the connections and configurations and tests with PON equipment. For the network infrastructure, passive optical network equipment was used, such as: OLT and ONT / ONU, service providers (Promax header, servers for VoIP and web), SIP telephones and computers receiving the services offered. Finally, the tests of reception of services in the ONT / ONU, were based on the official documentation of the manufacturer ZTE Corporation and of the company Promax, for the management of the provider header of the multicast transmission of IPTV. The approach proposed for the above is observed in Figure 1.

A.

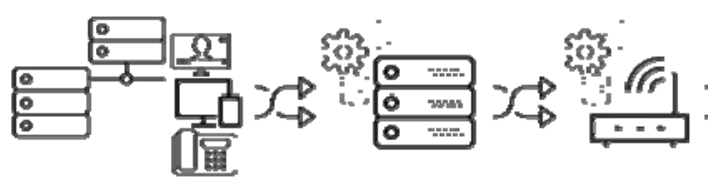

Fig. 1: Conceptual approach proposed
C.

D.

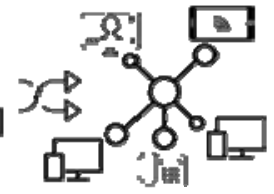

Before carrying out the stages of the scheme, you must have a network infrastructure correctly implemented, with standards and technical specifications up to date. The proposed approach is summarized as follows:

\section{A. Make connections of the equipment involved in the GPON network}

Connections are made from the service provider equipment to the OLT, then from the OLT to the ONU, and finally the receiving equipment of the end user is physically linked to the ONU, which results in an ODN.

\section{B. OLT configuration}

Technical procedures are applied based on the configuration of the transparent layer of the GPON network, that is, logical parameters are established so that the OLT allows the transmission of voice, data and video services.

\section{ONT / ONU configuration}

The ONT / ONU is configured remotely through the OLT.

\section{Service integrity checks}

Depending on the service, the appropriate receiving equipment is connected to the ONU via an Ethernet link.

The achievement of the previous steps consists of the development of experimental practices that consolidate a basic GPON network, which serves as a starting point to conceive a scalable optical network by means of a logical, technical and explicit basis, which allows the user to obtain a network infrastructure providing services. 


\section{III.RESULTS}

The guide was used in the laboratory of the telecommunications section of SENA, Cuatro Vientos, Cartagena-Colombia. The application of the stages, to obtain the implementation of a GPON network, is represented in Figure 2 and described below.

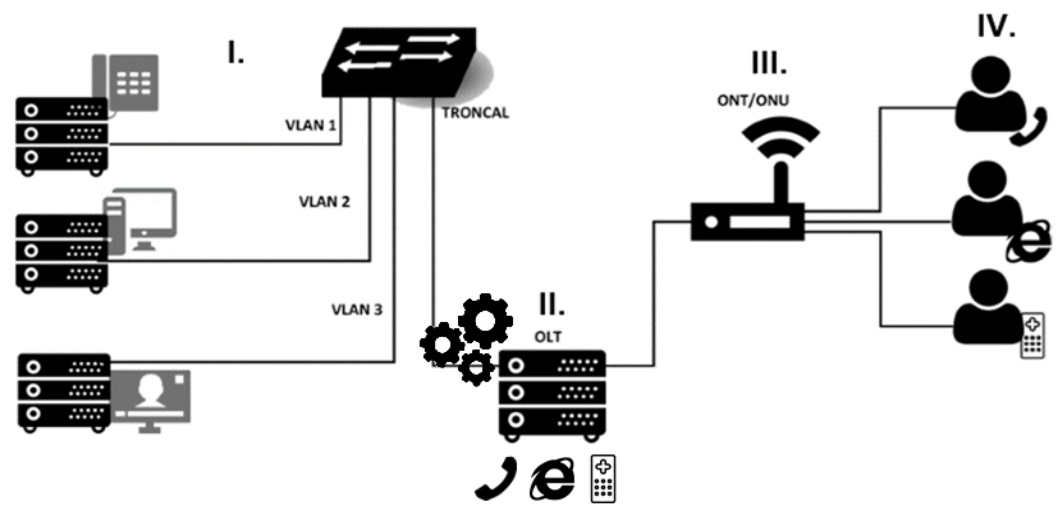

Fig. 2: GPON network implemented

\section{A. Make connections of the equipment involved in the GPON network}

The network infrastructures have a set of electronic devices that allow the flow of services, from their suppliers to the end user, for which this phase was subdivided into three parts:

1) Connection of the provider equipment to the OLT

Each service needs a provider source to carry out the transmission on the network. For the implementation of IPTV, a Promax header was used, which consists of configurable electronic modules that process the incoming signal (satellite, DTT, and / or multimedia as a DVD) for the multicast output; in the case of VoIP, an Elastix server was used, previously configured with accounts and extensions governed by the SIP protocol, installed in a virtual machine with an efficient Ethernet network connection; and in the case of the data service, an Apache server was used that hosted a web page on a connected computer.

Next, a switch was configured and VLANs was created for each service. Then, the provider teams connected to these, which distribute the services through the trunk port to the Gigabit / FastEthernet port of the OLT, as shown in section I of Figure 2.

\section{2) Connection of the OLT to the ONT / ONU}

The conditions of a network infrastructure type FTTC (FiberToTheCurb) were recreated, in which the outgoing optical fiber of the OLT, leaves a SC port connector to a cabinet that allows its ordering, connecting it with passive nodes such as the Mufa dome (having trays with fiber splices), until arriving at the point of optical distribution that contains splitters and attenuators that redistribute the section to one or more ONT / ONU.

\section{3) Connection of the service receiving equipment to the ONT / ONU}

The end user receives the service through the ONT / ONU, which has a PON port to execute instructions from the OLT through the fiber, in addition to Ethernet ports that allow connecting a device that processes the service, which are assigned from the OLT to receive specific services, that is, a LAN port of the ONT / ONU can be configured to transmit video (IPTV), voice (VoIP) or data (web service).

\section{B. OLT configuration}

This equipment must be configured according to the service transmitted, so it must conform to the corresponding protocol. Taking into account the above, we have the following cases, (section II, Figure 2):

1) Video service configuration

Being a high traffic service:

- $\quad$ A down and upload profile was configured (in this case $6000 \mathrm{Kbps}$ ).

- $\quad$ A high service priority was assigned.

- A VLAN was created analogous to the one configured on the switch, in which the provider equipment was connected.

- $\quad$ The main logical channel T-CONT was configured with its respective GEM port, which serves as an identifier of the multicast service, so that the ONT / ONU recognizes the GEM encapsulation frame that contains the video service. 
- The use of multicast IP transmission was enabled through the same VLAN through a logical interface.

2) Voice service configuration

Unlike the previous process, voice transmission requires less down and upload traffic (1000 Kbps in this case), since only audio bits and information about account authentications and SIP extensions flow. For the configuration of this service:

- A VLAN was configured analogous to the one established in the switch.

- A T-CONT, a GEM port and a voice port are configured (two Ethernet ports in this case) that connect the SIP phone to the ONT / ONU.

3) Data service configuration

Analogous to the previous configuration, many bits are not consumed in the download and upload profile (in this case $1024 \mathrm{Kbps})$, so:

- A VLAN similar to the one specified in the switch was established for the data provider team.

- A T-CONT specified for this service was established with its respective GEM port.

- An Ethernet port was configured for the ONT / ONU to receive the service.

The GEM encapsulation obtained as a result of the configuration of the OLT, is shown in Figure 3.

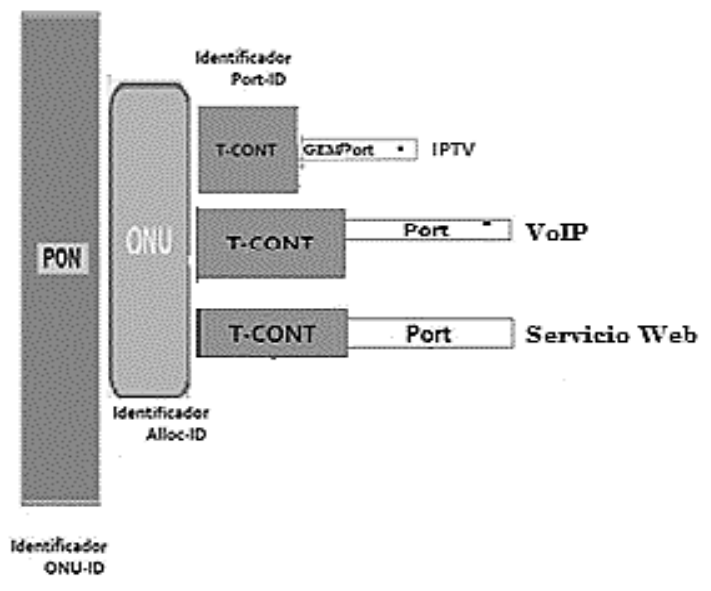

Fig. 3: GEM encapsulation resulting from the configuration

After configuring the OLT so that video, voice and data were transmitted, a GEM encapsulation was obtained that made it possible to assign logical channels for each service, which allowed an ONT / ONU to receive them properly.

\section{ONT / ONU configuration}

The link between OLT and the end user can be seen in section III of Figure 2. In this stage, a remote configuration was chosen from the OLT, after establishing the descent and upload profile with its priorities and GEM encapsulation containing the segments logical of the services to be transmitted. It is necessary for the ONU to recognize each service, so after enabling the remote configuration, each GEM port was assigned a name. It should be noted that, according to the protocol of the service, it was necessary to make corresponding configurations, such as:

\section{1) Video configuration}

A name was assigned to the GEM port and to the incoming VLAN in the OLT. Then, it is specified that the VLAN is multicast (MVLAN) and an Ethernet port is established in the ONT only to receive that service. Generally, this service is of high priority due to the demand for resources that it implies.

2) Voice configuration

A name was assigned to the GEM port with the corresponding VLAN and finally the Ethernet port for service reception was specified.

\section{3) Data configuration}

The process is similar to what was done previously: a name was assigned to the GEM port with its corresponding VLAN, in addition to an Ethernet port so that the service could be received properly. 
As additional data, more than one Ethernet port can be assigned to the ONT / ONU for service reception and priorities must be established, for example, services such as voice and data have lower priority compared to video.

\section{Service integrity checks}

After having configured each service from the OLT to the ONT / ONU, the process to check its integrity is as follows:

\section{1) Checking for video}

An IPTV receiver equipment was connected to the ONT / ONU Ethernet port, then the UDP protocol, IP and multicast port were entered in a multimedia player (in this case, VLC media player). With the steps previously carried out, the video reception was checked.

\section{2) Checking for voice}

The process was carried out:

- $\quad$ Connection of a SIP Phone to an Ethernet port on the ONT.

- $\quad$ Entry of an account with its respective password and SIP extension.

- $\quad$ IP assignment of the voice server (SIP Proxy), network mask and IP of the phone (it is necessary to highlight the need to be in the same network range).

In the same way, these configurations are made with another SIP Phone that will be connected to the network. This can be located on the switch side before the OLT or on another Ethernet port of the same ONT and there is communication between them. In addition, when installing a SIP Phone emulator on the server or computer (it can be Zoiper or Softphone), and when assigning a SIP account and extension, the reciprocal communication between a virtual SIP Phone with one or more physical SIP Phone was checked.

\section{3) Checking for data}

A computer connected to an Ethernet port of the ONT. Then, the client computer was configured in the same network range as the server and the address of the page hosted in a web browser was written, which consisted of the following syntax:

\section{SERVER_IP/WEB_PAGE_DIRECTORY/PAGE_FILE}

The transmission of data is checked at the moment in which the user can see the start of the page.

After checking the integrity of the services, they are suitable for use, as can be seen in section IV of Figure 2.

\section{E. Discussions}

The research, experimental and practical, have documentation according to the degree of knowledge that the public has on the subject. However, it was observed that the majority of academic and technical texts on GPON are for specialized public in the field of telecommunications. An example of the above, is the implementation guide for GPON under the standard G.984.4 [8], designed to clarify doubts and point out maintenance processes related to their interoperability, establishing guidelines for best practices; It is a document focused on specialists in fiber optic networks, and therefore, complex for public with little knowledge in networks and telecommunications.

Texts such as the [11], with specifications established when recommending an implementation or JAVA source code provided for testing and improvement; GPON as an alternative network infrastructure, by [12]; and [13], with proposals such as implementing a GPON network for transmission of triple play, confirm what was expressed in the previous paragraph, because they contain specialized topics and demand suitable users for that purpose.

It should be noted that the documentation generated in research regarding GPON is important for the growth of the telecommunications industry, which generates a social and economic impact in the world, as observed in the thesis on the analysis and impact of the incorporation of IPTV over a GPON network [14]; or the technical documentation of OLT and ONU equipment by manufacturers such as Cisco, FiberHome, ZyXEL, Huawei, ZTE, and others.

Taking into account the above, this guide is presented as a tool in the teaching of technical procedures for the implementation of a GPON network, which includes installation and configuration of its elements, by making available to users with little knowledge in telecommunications, a means to obtain a functional GPON infrastructure, which is a difference from the investigative, experimental and applied approach that currently exists. This guide includes an educational process that starts from the theoretical to the practical, which addresses the necessary components for the implementation of a GPON network. 


\section{IV.CONCLUSION}

According to the results obtained, it was possible to conclude:

- In the context of the city of Cartagena, it is difficult to develop a GPON network implementation that transmits the services implemented in the guide, due to the limited distribution of this type of network compared to technologies such as Ethernet, ADSL and HFC.

- The guide is a starting point for future work where complex network topologies are linked. The procedural education proposed in, it is important to understand how a GPON network works and how its components are connected and configured, in order to obtain a proven reception of services.

- $\quad$ The proven topologies are basic due to the equipment used to implement the implementations, but they show the bases to conceive a scalable network, which is left to freedom for future research applied in the field of GPON and its aspects.

\section{ACKNOWLEDGMENT}

Thanks to the collaboration of SENA, for the use of telecommunications facilities and equipment, it was possible to implement a GPON network that would independently transmit the services. In addition to providing documents for the use of commands to enter the OLT, which was helpful in the process of creating parameters.

\section{REFERENCES}

[1] ITU (2015). ICT Fact \& Figures. Ginebra: ITU. [Online] Available: https://www.itu.int/en/ITUD/Statistics/Documents/facts/ictfactsfigures2015.pdf

[2] Dane, P. and Kaushal, H. (2013). Characterization of RoF GPON Performance for Different Modulation Schemes, 2013 Tenth International Conference on Wireless and Optical Communications Networks (WOCN), 1-6.

[3] Cheng, H., Gao, J., Wu, H., Liu, G., Lau, E., Yuan, L. and Krause, C. (2016). Optics VS. Copper - From the Perspective of Thunderbolt 3 Interconnect Technology, 2016 China Semiconductor Technology International Conference (CSTIC), 1-3.

[4] Fowdur, T.P., Ragpot, P. and Soyjaudah, S.K.M. (2015). Enhanced audio transmission over ADSL using prioritized DMT modulation and retransmissions, IEEE EUROCON 2015 - International Conference on Computer as a Tool (EUROCON), 1-6.

[5] Zhu, Z. (2012). Design Green Hybrid Fiber-Coaxial Networks: A Traffic-Aware and Cooperative Approach, 2012 IEEE International Conference on Communications (ICC), 3165-3169.

[6] Kramer, G., De Andrade, M., Roy, R. and Chowdhury, P. (2012). Evolution of Optical Access Networks: Architectures and Capacity Upgrades, Proceedings of the IEEE, 100(5), 1188-1196.

[7] Lee, S., Li, K. and Wu, M. (2016). Design and Implementation of a GPON-Based Virtual Open Flow-Enabled SDN Switch, Journal of Light wave Technology, 34(10), 2552-2561.

[8] ITU-T (2008). G.984.4 Implementer’s Guide. Ginebra: ITU. Available: https://www.itu.int/rec/T-REC-G.Imp984.4-200812-S/en

[9] Martelo, R.J., Ponce, A.L. and Acuña, F. (2016). Guía metodológica para el diseño de un plan estratégico informático en instituciones de educación superior, Formación universitaria, 9(1), 91-98.

[10] Martelo, R.J., Villabona, N. and Jiménez-Pitre, I. (2017). Guía Metodológica para Definir el Perfil Profesional de Programas Académicos Mediante la Herramienta Ábaco de Régnier. Formación universitaria, 10(1), 15-24.

[11] Broadband Forum (2014). ATP-247 GPON \& XG-PON1 ONU Conformance Abstract Test Plan. Available: https://www.broadbandforum.org/technical/download/ATP-247_Issue-3.pdf.

[12] Cobos, V. (2011). Design and Performance Evaluation of Passive Optical Networks (Master Thesis). Universitat Politécnica de Catalunya. Catalonia: Escola d'Enginyeria de Telecomunicació i Aeroespacial de Castelldefels.

[13] Carmona, D., Prat, J., Tomkos, I. and Sánchez, M. (2012). GPON unbundling for multioperator access, In Telecommunications Network Strategy and Planning Symposium (NETWORKS), 2012 XVth International, IEEE, 1-6.

[14] Borja, C.A. and Peña, D. (2014). Análisis e impacto de la incorporación de IPTV sobre una red GPON (Proyecto de grado). Universidad Politécnica Salesiana. Cuenca, Ecuador.

\section{AUTHOR PROFILE}

Raul J. Martelo works as full-time professor at the University of Cartagena (Colombia). Mr. Martelo completed his magister from Industrial University of Santander (Colombia). Mr. Martelo completed his undergraduate in Systems Engineering at the Industrial University of Santander.

Hector Triana completed his undergraduate in Systems Engineering at the University of Cartagena (Colombia).

Gustavo Rodríguez completed his undergraduate in Systems Engineering at the University of Cartagena (Colombia). 